\title{
La modernisation de la justice comme pédagogie du changement : le cas de l'intégration de " conseillers en gestion des ressources humaines " dans les juridictions belges
}

Making justice modern by learning to change: the case of introducing human resources counselors in Belgian jurisdictions

Joël Ficet et David Delvaux

\section{OpenEdition} Journals

Édition électronique

URL : http://journals.openedition.org/pmp/2201

DOI : $10.4000 / p m p .2201$

ISSN : $2119-4831$

Éditeur

Institut de Management Public (IDPM)

Édition imprimée

Date de publication : 15 mars 2010

Pagination : 55-74

ISSN : 0758-1726

Référence électronique

Joël Ficet et David Delvaux, «La modernisation de la justice comme pédagogie du changement : le cas de l'intégration de " conseillers en gestion des ressources humaines » dans les juridictions belges », Politiques et management public [En ligne], Vol 27/2 | 2010, document 27, mis en ligne le 15 mars 2012, consulté le 08 mai 2019. URL : http://journals.openedition.org/pmp/2201 ; DOI : 10.4000/ pmp.2201 


\title{
LA MODERNISATION DE LA JUSTICE COMME PEDAGOGIE DU CHANGEMENT : LE CAS DE L'INTEGRATION DE « CONSEILLERS EN GESTION DES RESSOURCES HUMAINES » DANS LES JURIDICTIONS BELGES
}

Joël FICET ${ }^{1}$

David DELVAUX ${ }^{1}$

\begin{abstract}
Résumé Les tribunaux belges viennent de connaître une double mutation : la dépolitisation de la magistrature d'une part, l'amélioration de l'efficience du service public d'autre part. Parmi l'arsenal des moyens mis en œuvre, l'article s'attachera à analyser une des mesures: l'insertion à titre expérimental de « conseillers en gestion des ressources humaines » dont l'objectif principal est de réduire progressivement le délai de traitement des affaires. Mais l'impact de l'introduction de ce nouveau corps semble très marginal quant à son objectif officiel. En revanche, cet élément de réforme a contribué à un changement de nature cognitive, ouvrant la voie à une appropriation des méthodes managériales par les acteurs du monde judiciaire grâce au nouveau cadre de délibération qu'il a permis d'introduire.
\end{abstract}

Mots clés Belgique, changement, GRH, justice, modernisation, service public

\begin{abstract}
Belgium courts has just gone through a double change: the magistracy's depoliticization on one hand, the improvement of public service's efficiency on the other hand. Among the lot of implemented measures, this article focuses on the insertion in the place, experimentally, of "counsellors in human resources' management" with the principal objective to reduce progressively the treatment's time of affairs. But the impact of this introduction seems to be marginal as for its official goal. Meanwhile, this reform's action has contributed to a cognitive change, has opened a way to managerial methods' appropriation by judicial actors thanks to the new space of deliberation created.
\end{abstract}

Keywords Belgium, human resources' management, justice, modernisation, public service

\footnotetext{
${ }^{1}$ CRIS - Université de Liège

Revue POLITIQUES ET MANAGEMENT PUBLIC, Volume 27, n² 2, 2010.

(C) Institut de Management Public - 2010.
} 
Introduction
Les tribunaux belges sont depuis une décennie le théâtre de profondes mutations. Le point de départ de cette vague réformatrice est généralement situé au lendemain de l'affaire Dutroux (Sancken, 2001), qui a révélé au grand public les dysfonctionnements de l'appareil pénal et inscrit la rénovation de la Justice à l'agenda politique (Vigour, 2004). Un consensus se dégage alors au sein de la classe politique pour une «modernisation " forcée de l'institution judiciaire, consensus matérialisé initialement par l'accord Octopus (surnommé ainsi car appuyé par les huit principaux partis de Belgique) du 24 mai 1998 (Laenens, Storme, 2000). Cette modernisation a en fait deux dimensions : la dépolitisation de la magistrature d'une part, l'amélioration de l'efficience du service public de la Justice d'autre part.

Le premier objectif est à l'origine de la création du Conseil supérieur de la Justice (CSJ), composé pour moitié de magistrats élus par leurs pairs et de représentants de la société civile, et chargé de contrôler le recrutement et l'avancement des membres du corps judiciaire ; l'intervention de cet organe a permis dans une large mesure de couper le cordon entre classe politique et corps judiciaire (Verdussen, 1999). L'effort réformateur s'est depuis lors essentiellement porté sur la question de la performance des tribunaux et sur la transposition en leur sein de la philosophie et des méthodes de la Nouvelle Gestion Publique (Gow, Dufour, 2000 ; Hood, 1991 ; de Visscher, Varone, 2004). Ainsi, dans sa note de politique générale, l'actuel Premier ministre se donne pour objectif la promotion d'une " justice performante, moderne et soucieuse de résultats ${ }^{2}$. En avril 2008 , le ministre de la Justice fait lui aussi état de son ambition en ce sens, en insistant sur le développement d'une Justice qui doit suivre « la tendance générale de la transparence, de l'efficacité et de l'effectivité, au même titre que les entreprises du secteur privé et les institutions publiques ${ }^{3}$. L'objectif de l'insertion dans le système judiciaire d'une culture managériale est donc clairement affiché.

Pour mener à bien ce processus, un arsenal de moyens de différentes natures a été développé : structurels (mise en place de nouveaux organes comme le CSJ ou la Commission de Modernisation de l'Ordre judiciaire), fonctionnels (outil de mesure de la charge de travail, cercles de qualité, intégration des bases de données judiciaires...) et humains (responsabilisation des chefs de corps, formation des juges et greffiers au management...). Un ambitieux programme de décentralisation de la gestion des juridictions (Plan Thémis) a également été annoncé par le gouvernement Verhofstadt en 2003; la crise politique présente en a néanmoins gelé la mise en application.

L'objectif de ce texte, élaboré dans le cadre d'un programme de recherche sur les réformes judiciaires en Belgique mené au sein du Centre de Recherche et d'Intervention Sociologiques (CRIS) de I'Université de Liège, est de rendre compte des aléas de cette entreprise de transformation des pratiques gestionnaires de la Justice au travers de l'analyse d'une des mesures issues de la politique de modernisation: l'insertion à titre expérimental de « conseillers en gestion des ressources

\footnotetext{
${ }^{2}$ Note gouvernementale du 16 octobre 2007, chapitre 6, p.54.

${ }^{3}$ Déclaration de Politique Générale du Ministre de la Justice Jo Vandeurzen, avril 2008, p.57.
} 
La modernisation de la justice comme pédagogie du changement : le cas de l'intégration de "conseillers en gestion des ressources humaines " dans les juridictions belges

humaines » dans un certain nombre de parquets et de juridictions belges. La création de ce nouveau statut est prévue par des "protocoles d'accord " entre le ministère de la Justice et les chefs des corps concernés. Le premier de ces protocoles a été signé en mars 2004 par la ministre de la Justice de l'époque, Laurette Onkelinx, et le Premier président de la Cour d'appel de Bruxelles. Leur objectif est en apparence simple : il s'agit de " dégager de part et d'autre les moyens nécessaires pour réduire progressivement le délai de traitement des affaires et lutter ainsi contre l'arriéré judiciaire ». Pour ce faire, les protocoles prévoient tout d'abord une extension du cadre des magistrats et un renfort du personnel de greffe, mais aussi, sur la base des recommandations formulées par le CSJ en faveur d'une " politique moderne des ressources humaines ", la création de postes de conseillers en gestion des ressources humaines $(\mathrm{GRH})$. Ces derniers se voient chargés d'assurer un meilleur emploi des ressources humaines disponibles et d'appuyer le Premier président en ce qui concerne la répartition du personnel judiciaire et administratif, la distribution des tâches, l'adaptation des processus de travail, la motivation des personnels et la communication interne. Son domaine officiel d'intervention englobe donc l'ensemble des aspects de la politique de modernisation dont il apparaît, à de nombreux égards, comme l'incarnation et le représentant dans les juridictions.

Cinq corps ont à l'heure actuelle signé de tels protocoles : la Cour d'appel et le Parquet de première instance de Bruxelles, la Cour d'appel et le Parquet général de Liège et la Cour d'appel d'Anvers. Chacun des conseillers présents dans ces instances a été interrogé sur les étapes de son insertion, sur la nature des tâches qu'il est amené à remplir, sur ses rapports avec les autres acteurs de la juridiction ainsi que sur ses contacts avec des agents extérieurs potentiellement intéressés à leur action (cabinet du ministre, Conseil supérieur de la Justice, autres conseillers...). Des entretiens ont également été réalisés auprès de leurs chefs de corps et d'autres acteurs de leurs juridictions sur les mêmes thèmes. Enfin, ont été rencontrés les initiateurs du dispositif au sein du cabinet de la ministre de la Justice d'alors.

L'ambition première de cette étude était d'analyser, dans une perspective de sociologie des organisations, l'impact de ce nouveau dispositif sur les " ordres locaux" établis au sein de chaque corps (Friedberg, 1993); il était implicitement postulé que l'apparition d'un nouvel acteur au cœur des processus de gestion devait se traduire par une réévaluation des rôles, des stratégies et des pratiques de travail propres au système d'action considéré. Or, l'observation ne révèle au premier abord qu'une influence très marginale des conseillers sur le fonctionnement au quotidien des organisations auxquelles ils sont rattachés : pour la plupart isolés au sein de leur juridiction, ils n'ont généralement de contacts avec les magistrats qu'au travers du chef de corps. La liste de leurs compétences, constamment redéfinie au gré des besoins et circonstances, ressemble avant tout à un catalogue des tâches dont personne ne veut. Enfin, leurs initiatives les plus ambitieuses - celles qui touchent aux processus de travail - restent le plus souvent lettre morte. Les promoteurs de l'innovation au niveau ministériel ne se déclarent d'ailleurs guère étonnés de ce résultat: conscients de la résistance des 
structures de l'institution judiciaire au changement, ils déclarent avoir conçu les conseillers avant tout comme des vecteurs de diffusion d'une nouvelle culture professionnelle. Notre hypothèse est que leur impact doit dès lors être analysé au regard de cet objectif de transformation des valeurs et schémas de pensée des personnels de la Justice.

On se positionne là dans une perspective de sociologie de la gestion (Berry, 1983 ; Maugéri, 2006 ; Metzger et Benedetto-Meyer, 2008) qui souligne les dimensions inséparablement matérielles, humaines et symboliques des dispositifs managériaux. Ces derniers doivent en effet être appréhendés non seulement comme des agencements de ressources techniques (règles de droit, applications informatiques, indicateurs...) au service d'une performance organisationnelle définie dans l'abstrait, mais au travers des interactions qu'ils génèrent et des significations qu'ils cristallisent. II devient alors possible de mettre en avant les fonctions implicites de l'introduction de ce nouvel instrument, qui a pour objet tant de modifier effectivement les pratiques des greffiers et magistrats que de propager et légitimer auprès d'eux les normes propres au référentiel managérial; ils constituent ainsi des instruments de socialisation dont les effets s'observent avant tout dans la façon dont les acteurs perçoivent et débattent des buts et problèmes de l'organisation à laquelle ils appartiennent.

Cette interprétation s'inscrit dans une conception large du changement organisationnel, inspirée par Denis Segrestin. Celui-ci postule qu'une action produit "des apprentissages dignes de considération si l'appropriation qui en est faite sur le terrain redéfinit l'espace de discussion à l'intérieur duquel les acteurs règlent les problèmes qu'ils affrontent » (2007, p. 28) ; les innovations managériales fonctionnent alors comme des "opérateurs cognitifs" en transformant les représentations de l'organisation et en contribuant à construire ce que Claude Michaud et Jean-Claude Thoenig nomment un nouveau « langage pour l'action » (Michaud, Thoenig, 2001). Cette approche est éclairante quant aux finalités et conséquences de l'introduction de ces conseillers en $\mathrm{GRH}$, mais aussi d'autres outils récemment développés dans la Justice belge et également étudiés par notre équipe (mesure de la charge de travail des magistrats, démarches qualité dans les parquets...): dans l'impossibilité d'imposer des réformes brutales à un corps judiciaire riche de ses traditions et constitutionnellement indépendant, le politique a favorisé des dispositifs plus discrets destinés à familiariser les juges avec d'autres méthodes et raisonnements, ainsi qu'à susciter leur adhésion à des principes de maîtrise des coûts, de rationalisation de l'usage des ressources, de proactivité, de qualité du service, etc.

Ces considérations conduisent à une analyse à deux niveaux de cette innovation. En un premier temps, on présentera la genèse de ce dispositif et la difficile insertion des conseillers dans des organisations hiérarchisées, formalistes et composées de professionnels défendant jalousement leur autonomie. Le constat de ce bilan en demi-teinte nous amènera à affiner l'analyse en mettant en un second temps l'accent sur la dimension cognitive de cet instrument d'une politique de modernisation qui trouve sa cohérence, comme on le montrera à l'aide de quelques exemples issus d'autres recherches poursuivies par notre centre, dans 
La modernisation de la justice comme pédagogie du changement : le cas de l'intégration de "conseillers en gestion des ressources humaines » dans les juridictions belges

une volonté affirmée de transformation des mentalités des acteurs de la Justice. Ce micro-dispositif est donc ici interprété comme symptomatique de la façon dont est mené en Belgique et depuis dix ans le processus de modernisation de l'ordre judiciaire.

Du cabinet ministériel aux juridictions : genèse et mise en œuvre d'un dispositif managérial dans une organisation rétive au changement
La création des conseillers s'insère dans un mouvement plus large de rationalisation de la gestion des ressources humaines et financières des tribunaux en Belgique. L'attribution et la répartition des cadres de magistrats dans les différentes juridictions du pays sont traditionnellement davantage basées sur les affinités et la qualité des relations entre chefs de corps et cabinet ministériel que sur une analyse standardisée et objective des besoins réels des juridictions. Ce modus operandi ne pouvait évidemment que générer des tensions clientélistes au sein de la sphère judiciaire et affecter la qualité des prestations des tribunaux. La problématique de l'attribution des postes de magistrats s'est donc naturellement imposée comme un des enjeux majeurs de la réforme de l'Etat engagée. En témoigne le conditionnement de toute extension des cadres à une mesure objective de la charge de travail des juges, affirmé dès 2000 par un courrier du ministre de la Justice de l'époque aux Premiers présidents des Cours d'appels du Royaume : " l'arriéré judiciaire constitue une de mes préoccupations prioritaires, néanmoins toute demande d'extension du cadre requiert une justification minutieuse sur la base de la mesure de la charge de travail ». Les protocoles d'accord entre chefs de corps et ministère sont en grande partie les conséquences de la difficulté à mettre en place un instrument objectif et consensuel de mesure de la charge de travail, bien que son existence soit prévue depuis 2001 par le Code judiciaire ${ }^{4}$.

En effet, confronté à de nouvelles demandes d'extension de cadre formulées par différents chefs de corps en 2005, le cabinet ministériel entend néanmoins ne pas procéder à une attribution non maîtrisée de magistrats supplémentaires. Les protocoles constituent donc des contrats d'engagement réciproques et négociés entre le cabinet de la ministre et ces chefs de corps. Le marché conclu se résume ainsi: le Ministère s'engage à étendre les effectifs de magistrats et du personnel de greffe des juridictions à la condition que celles-ci s'emploient activement à diminuer l'arriéré judiciaire et acceptent l'apport d'un conseiller en ressources humaines. Du point de vue des chefs de corps, l'introduction d'un conseiller apparaît donc avant tout comme une concession faite au pouvoir politique. II n'est dès lors guère étonnant que ces nouveaux acteurs se soient heurtés à une certaine hostilité et qu'ils aient dû négocier graduellement leur rôle au sein des juridictions. Cette première partie s'attache à décrire le processus d'intégration des conseillers en $\mathrm{GRH}$, en partant de l'élaboration du dispositif et des polémiques qui ont l'entouré jusqu'à leur "parachutage » sur le terrain et la conquête progressive d'une légitimité à s'exprimer sur la gestion de leur corps d'affectation d'abord, à y intervenir ensuite.

\footnotetext{
${ }^{4}$ L'article 352bis du Code judiciaire stipule que « le roi détermine, après avis du Conseil Supérieur de la Justice, la manière dont est enregistrée la charge de travail du juge et du ministère public ainsi que la manière dont ces données enregistrées sont évaluées ».
} 


\section{Les conseillers en gestion des ressources humaines, enjeux de pouvoir entre cabinet ministériel et chefs de corps}

La réaction première des chefs de corps à l'initiative du cabinet ministériel s'articule autour de deux enjeux: l'atteinte potentielle au principe d'indépendance de la Justice, et le positionnement hiérarchique des conseillers à l'égard des acteurs en place.

De fait, le principe d'indépendance, consacré par la Constitution en ses articles 151, 152,154 et 155, cristallise la majorité des débats liés à l'insertion de dispositifs managériaux dans les cours et tribunaux. Sur le plan symbolique, il met en exergue la fonction particulière de la Justice dans l'ordre démocratique et matérialise sa spécificité vis-à-vis des autres segments de l'Etat. Mais, au-delà de ce rôle de légitimation de l'institution, il contribue à garantir la relative autonomie professionnelle dont bénéficient les magistrats. La structuration des systèmes d'action juridictionnels, appuyée sur des arrangements négociés plus que sur l'exercice direct de l'autorité hiérarchique, assure à chaque juge la maîtrise de ses méthodes et préserve à son profit une marge de manœuvre irréductible - même par le chef de corps ${ }^{5}$.

En cela, les juridictions ressemblent aux «bureaucraties de professionnels " telles que les universités ou les hôpitaux (Mintzberg, 1990, pp. 255-284). Or, le management moderne repose dans une large mesure sur l'objectivation et la normalisation des conventions d'action au sein des organisations; il suppose par ailleurs une responsabilité gestionnaire (accountability) des acteurs. La transparence et la standardisation des processus de travail impliquées par les dispositifs managériaux ne peuvent dès lors que réduire la "zone d'incertitude » (Crozier, Friedberg, 1992, pp. 71-73) ouverte aux magistrats (et, dans une moindre mesure, au personnel administratif) par le fonctionnement relativement informel et opaque de l'organisation, et qui leur permet de négocier le degré et la forme de leur investissement dans les tâches judiciaires. Les réticences voire l'agressivité constatées des praticiens face à une telle perspective ne sont donc pas entièrement incompréhensibles. L'invocation du principe d'indépendance apparaît souvent dans cette perspective comme constitutive d'une stratégie de résistance au changement et à l'immixtion de l'exécutif dans les affaires de la Justice que représenterait, selon eux, le déploiement du management dans les juridictions.

Les conseillers en gestion des ressources humaines n'ont bien évidemment pas échappé à cette méfiance. En l'occurrence, les magistrats redoutaient que ces « experts » de l'extérieur ne deviennent des outils de contrôle au service du ministère (" l'œil de Moscou », pour reprendre une formule souvent utilisée par les conseillers eux-mêmes

\footnotetext{
${ }^{5}$ Ceci est surtout vrai au siège, les magistrats du ministère public étant placés plus directement sous l'autorité hiérarchique des procureurs et, au-delà, de l'administration centrale de la Justice. Toutefois, même dans les parquets, les interventions dans les dossiers individuels ou dans les pratiques des juges sont rares.
} 
La modernisation de la justice comme pédagogie du changement: le cas de l'intégration de "conseillers en gestion des ressources humaines " dans les juridictions belges

pour décrire la méfiance des juges à leur égard). Certaines des tâches qui leur sont affectées (établissement de statistiques, audit dans les services administratifs, contribution à la rédaction des rapports annuels de juridiction) pouvaient en effet être interprétées comme une forme de surveillance des activités individuelles et collectives des personnels.

"Il y a des magistrats qui n'ont pas voulu [d'un conseiller en gestion des ressources humaines]. Pourquoi ? C'est toujours cette méfiance intrinsèque: c'est l'œil de la ministre de la Justice dans le fonctionnement de la Cour. Non, il est sous mon autorité. II fait un rapport annuel qu'il rend à la ministre mais elle ne viendra jamais lui dire 'dites leur de faire comme ça ou comme ça'. Mais il y a toujours cette méfiance ancestrale entre les juges, les gouvernants et le législateur » (entretien, Premier Président de Cour d'appel).

Un autre ordre d'inquiétude concernait la place des conseillers dans l'organigramme des juridictions. Deux modalités ont à cet égard été envisagées au cours de la négociation. La première était de former au sein du Ministère de la Justice, un pool mobile de conseillers en GRH. Dans cette configuration, préconisée par le cabinet ministériel, les conseillers seraient installés dans les juridictions concernées mais, ultérieurement, pourraient être envoyés dans d'autres tribunaux pour des missions ponctuelles. Une équipe de spécialistes externes, placée sous l'autorité du Ministère de la Justice, aurait ainsi été constituée. L'alternative consistait à intégrer les conseillers dans la hiérarchie de la juridiction, et ce sous l'autorité des chefs de corps. C'est cette approche, conforme aux souhaits de ces derniers, qui a en définitive été retenue.

Une telle décision doit également être interprétée à la lumière du débat sur le Plan Thémis mentionné plus haut. L'objectif essentiel de ce plan est la décentralisation de la gestion des budgets des juridictions (Pyramides, 2006). Les premières notes de réflexion élaborées dans cette perspective envisageaient la création de " managers en ressources humaines » et de «managers budgets" dans chaque ressort de Cour d'appel; ces managers auraient occupé, à l'indignation de nombreux magistrats, un niveau comparable à celui des chefs de corps dans la hiérarchie de l'institution (et, afin d'attirer des spécialistes du secteur privé, auraient probablement bénéficié de rémunérations supérieures) (Depré, Hondeghem, Plessers, 2008). Les craintes exprimées par le corps judiciaire sur ce sujet pendant l'élaboration du Plan Thémis (et qui ont contribué à son enlisement) ne pouvaient qu'être ravivées par l'annonce de la création de "conseillers en gestion des ressources humaines ", possibles chevaux de Troie d'un dessaisissement des prérogatives managériales des procureurs et présidents de juridiction; le fait de les mettre ostensiblement en position de simples assistants des chefs de corps et de les priver de toute autorité effective est pour le cabinet ministériel un moyen de faciliter leur insertion professionnelle.

Une telle décision n'est pas sans conséquences : elle permet aux chefs de corps de canaliser et contrôler étroitement le travail réalisé par le conseiller en ressources humaines. En effet, le conseiller ne dispose d'aucun pouvoir contraignant: tous les initiatives et dispositifs qu'il 
souhaiterait développer pour améliorer la gestion et les processus de travail au sein de la juridiction nécessitent l'accord en aval du chef de corps sous l'autorité duquel il est placé. Ce choix explique également le relatif isolement des conseillers vis-à-vis des autres acteurs de leur corps et leur difficulté à y conquérir un rôle légitime.

\section{La difficile intégration des conseillers dans les juridictions}

\section{a. Des acteurs marginalisés}

La description faite par chaque conseiller de l'accueil qui lui a été fait au sein de son corps d'affectation diffère. Dans son premier rapport de fonctionnement, le conseiller placé auprès de la Cour d'appel de Bruxelles affirme que son arrivée a été "très bien préparée "; à l'inverse, son collègue de la Cour d'appel de Liège évoque en entretien une certaine indifférence. Mais les témoignages convergent pour déplorer, dans le cadre de leur travail quotidien, un certain isolement vis-à-vis des magistrats - hormis le chef de corps et, éventuellement, ses plus proches adjoints. Les conseillers éprouvent en effet de grandes difficultés à se situer au sein d'une institution caractérisée par une division rigide du travail entre trois groupes communiquant peu et disposant de modes de régulation spécifiques: les magistrats du siège, ceux du parquet et le personnel administratif.

Deux dimensions à cet isolement peuvent être distinguées. La première est fonctionnelle. Les conseillers sont les seuls à travailler dans le champ des ressources humaines. Leur seul interlocuteur est le chef de corps, en charge de l'organisation de sa juridiction. Or, si leurs responsabilités se chevauchent, ces deux acteurs conçoivent généralement la gestion des ressources humaines en des termes diamétralement opposés. Les conseillers, formés aux techniques managériales, la pensent en termes d'emploi optimal des ressources existantes et d'évaluation rationnelle des besoins en personnel à l'aide d'outils et de procédures standardisés (profilage de fonction, audits, tableaux de bord...), alors que le chef de corps privilégie une gestion de "bon père de famille ", basée sur la connaissance des réalités du terrain et des acteurs magistrats qu'il dirige. Ce dernier mode de gestion apparaît ainsi comme contingent et " humain » dans la mesure où les chefs de corps déclarent souvent que la connaissance personnelle des magistrats, de leur capacité de travail, de leurs préférences et spécialisations dans certains domaines, voire même de leurs réalités familiales, permet un fonctionnement harmonieux et efficace de la juridiction (Schoenaers, 2003 ; Ficet, 2008a).

La seconde dimension de cet isolement est culturelle. Les conseillers en GRH sont en quelque sorte les victimes d'un antagonisme plus général entre la culture professionnelle de la magistrature et les valeurs managériales. Cette culture ne se résume en effet pas à des méthodes de travail et repose, comme le montre Cécile Vigour, sur la croyance en la spécificité de la Justice parmi les autres pans de l'Etat. Selon celle-ci, l'" éthos professionnel " de la magistrature est structuré autour de quatre éléments, qui tous soulignent la singularité de l'institution judiciaire et, conséquemment, l'inadéquation des recettes du management public à sa réforme. Ces éléments sont: l'autonomie et l'indépendance des 
La modernisation de la justice comme pédagogie du changement : le cas de l'intégration de "conseillers en gestion des ressources humaines " dans les juridictions belges

magistrats dans le cadre de leurs activités juridictionnelles, que l'on a déjà soulignée plus haut; le statut particulier et transcendant du droit, notamment comme vecteur de liberté; la valorisation de la rigueur technique, de l'individualisation des dossiers, de la pondération (« l'éloge de la lenteur $»)$; l'incapacité à penser l'institution judiciaire comme une organisation (2006, p. 427).

Ces dispositions apparaissent évidemment incompatibles avec les principes les plus établis du management public, dont les conseillers sont censés être les porte-parole : application des méthodes du secteur privé dans les administrations publiques, accountablity, évaluation par les résultats (par opposition à une évaluation comme vérification du respect des procédures), standardisation des méthodes de travail... Les recommandations des conseillers sont dès lors souvent remises en cause du simple fait de leur assimilation à une logique managériale perçue par les juges comme menaçante pour leur identité professionnelle.

\section{b. La conquête d'une légitimité et la négociation d'un rôle}

Ces éléments contribuent à placer les conseillers en marge du système d'action juridictionnel. Dans ces conditions, la question de la fonction et du rôle effectif (en opposition avec le rôle formellement prévu au sein des protocoles d'accord) des conseillers interpelle. Placés sous l'autorité du chef de corps, leur autonomie est limitée: "La fonction, c'est juste de donner des conseils. On n'est nulle part dans la ligne hiérarchique. On est en staff, on soutient le chef de corps et le responsable administratif. (...) C'est un gros frein, parce qu'il faut toujours qu'on soit suivi pour qu'il se passe quelque chose. On n'a pas de pouvoir proprement d'initiative sur le terrain, donc ça c'est un gros frein pour insuffler une vraie dynamique de gestion " (entretien, conseiller en GRH). In fine, elle dépend donc de la capacité du chef de corps à imaginer une alternative à la gestion actuelle des juridictions - la gestion en bon père de famille -, de sa sensibilité personnelle aux principes de la Nouvelle Gestion Publique et de sa compréhension des exigences managériales qui la caractérisent.

De ce fait, la première tâche du conseiller consiste à négocier son rôle au sein du système d'action juridictionnel, principalement auprès du chef de corps. Quelles seront ses missions concrètes et dans quels domaines de gestion du corps s'opéreront-elles? Pour certains conseillers, la démarche va même plus loin : il s'agit tout d'abord de convaincre le chef de corps du bien-fondé de leur présence et des perspectives qu'elle offre. Rappelons ici que si certains chefs de corps étaient relativement enthousiastes quant à l'apport d'un conseiller, pour d'autres cet appui s'apparente davantage à une concession nécessaire pour l'obtention de magistrats supplémentaires. Dans ce cas de figure, c'est à une véritable opération de séduction que doit se livrer le conseiller à l'égard du chef de corps.

II n'est dans ces conditions guère étonnant que la définition des tâches confiées à chaque conseiller au sein de son corps reste à l'heure actuelle, dans une large mesure, de l'ordre de l'informel. La dévolution des responsabilités à ceux-ci ne procède en effet pas d'un cahier de charges 
préalablement établi par le ministère ou le chef de corps, mais de nécessités circonstancielles ou d'initiatives personnelles :

"Ce conseiller en ressources humaines, pour moi, je l'ai vraiment utilisé à toutes les sauces" (entretien, Premier président de Cour d'appel)

" A nous vraiment de modeler notre fonction (...) II faut trouver son travail, en fait. Au début, il faut se lancer, trouver ses projets, et faire des propositions pour avoir du boulot, vu qu'il n'y a pas de suivi au niveau du SPF et qu'il n'y a pas de consignes du type 'vous devez faire une évaluation de la charge de travail, vous devez travailler sur tel ou tel domaine'. On fait ce qu'on veut » (entretien, conseiller en ressources humaines).

Leur domaine de compétences s'apparente donc à un catalogue disparate et hétérogène comprenant, d'une part, ce que l'on pourrait appeler le "dirty job » (Hughes, 1958), un ensemble de corvées que le personnel administratif refuse ou n'est pas à même de remplir (établissement de statistiques, rédaction des rapports de juridiction, animation de réunions d'information internes, relations publiques...), d'autre part, la mise en œuvre des quelques projets dont ils auront su démontrer la pertinence au chef de corps. La réalité de l'action des conseillers reste donc largement en-deçà des ambitions des protocoles, qui évoquaient une réflexion sur les processus de travail judiciaires en vue d'une réduction des arriérés.

De fait, confrontés à la réticence des magistrats à l'objectivation de leurs usages professionnels et de leur statut (heures de travail, congés, etc.), ils ont été conduits à se pencher essentiellement sur les conditions de travail du personnel administratif. Sur ce plan, les innovations imaginées ou mises en œuvre par les uns et les autres se ressemblent. La plupart touche la gestion des ressources humaines au sens classique du terme: élaboration de critères de sélection pour les catégories d'agents dont le recrutement ne dépend pas du ministère (vigiles, agents de nettoyage...), organisation de journées d'intégration pour les nouveaux arrivants, élaboration de grilles d'évaluation, formalisation de l'attribution des congés ou des tours de garde, description de fonctions...

Les conseillers peuvent également être amenés à effectuer des médiations en cas de conflits personnels. Sans pour autant assumer la fonction de véritables directeurs des ressources humaines, ils se sont donc imposés comme interlocuteurs des employés non-magistrats en tout ce qui concerne leur statut, au risque parfois d'entrer en concurrence avec le supérieur officiel de ces derniers, greffier en chef (au siège) ou secrétaire en chef (au parquet). Toutefois, l'envergure de ces interventions reste mineure, les fonctionnaires du SPF Justice conservant l'essentiel des compétences en matière de ressources humaines. L'apport des conseillers à la réforme des processus de travail des agents administratifs est par ailleurs fort réduit: rédaction de templates, élaboration de lettres-types (parquet de Bruxelles), éventuellement diagnostics organisationnels dans les services administratifs (parquet de Bruxelles, Cour d'appel de Liège), réorganisation du personnel de 
La modernisation de la justice comme pédagogie du changement : le cas de l'intégration de "conseillers en gestion des ressources humaines " dans les juridictions belges

sécurité et d'entretien, amélioration de l'accueil des nouveaux magistrats (Cour d'appel d'Anvers)... II s'agit donc là de micro-management, rationalisant à la marge les pratiques gestionnaires de l'organisation sans modifier les structures de celle-ci.

En ce qui concerne les aspects de leur mission qui touchent directement l'activité des magistrats, les conseillers disposent encore moins d'autonomie. Les chefs de corps, comme en témoigne un conseiller, considèrent en effet cette dimension de l'organisation comme leur territoire propre: "Le Premier président il a son territoire, il a ses magistrats, et il pense que c'est lui qui peut parler avec, et donc il me laisse des choses purement exécutives. Et donc s'il veut sélectionner des magistrats il me demandera de faire des résumés des dossiers des candidats, comme il n'a pas le temps de le faire. Mais bon ça ne change pas le monde judiciaire, ces choses... ". C'est donc ici l'impulsion du chef de corps et sa capacité à entraîner ses pairs qui déterminent l'agenda des éventuels changements. Le conseiller susnommé a ainsi en vain tenté de convaincre son supérieur de l'utilité d'un formulaire standardisé pour les entretiens d'évaluation des magistrats.

A contrario, l'autorité plus grande des procureurs sur leurs subordonnés semble avoir favorisé l'intervention de la conseillère du parquet de Bruxelles dans le monde des magistrats ${ }^{6}$. Elle a notamment été amenée à la demande du Procureur du roi à systématiser les règles d'attribution des congés et des obligations de garde des magistrats. L'innovation peut sembler anodine; néanmoins, dans un milieu professionnel opaque régi par des normes implicites de sociabilité, l'objectivation des routines et arrangements institutionnels par un acteur extérieur au corps est mal acceptée: "Cette semaine-ci, on sort une note sur les congés et les absences. A combien de jours de congés est-ce qu'on a droit, quelles sont les règles en la matière, etc. C'est toute une série de choses qui en soi n'ont pas l'air compliqué, mais c'est l'objet de questions voire de tensions, c'est... ça les tient très à cœur. Et donc c'est dangereux. C'est potentiellement explosif. Avec la population de magistrats, il faut être sûr de ce qui est envoyé ". Le constat d'une relative impuissance des conseillers concernant les processus de travail judiciaires ne doit toutefois pas être hâtivement érigé en règle générale. On peut ainsi citer en contreexemple une expérience de "démarche qualité " menée au cours de l'année 2006 à l'initiative du conseiller de la Cour d'appel de Bruxelles, et qui a débouché sur un rapport contenant deux cents propositions d'amélioration ; celles-ci sont toutefois pour l'instant restées inappliquées.

II apparaît à l'issue de cette analyse de l'élaboration des protocoles d'accord - dont les enjeux organisationnels ont été soulignés - et de l'intégration des conseillers en ressources humaines dans leur corps caractérisée par l'isolement, l'absence de pouvoir décisionnel et l'ambiguïté des missions effectivement assumées - que l'impact concret de ce dispositif managérial sur les processus de travail judiciaires est

\footnotetext{
${ }^{6}$ Le ministère public apparaît en effet de manière générale comme un terreau plus favorable aux réformes managériales, tant du fait de son organisation hiérarchisée que de sa plus grande ouverture aux partenaires extérieurs (police, municipalités, services sociaux...).
} 
limité. La pertinence de cette innovation doit toutefois, comme nous proposons de le faire en une seconde partie, être appréciée de manière plus large au niveau des représentations professionnelles.

Les

conseillers en gestion des ressources humaines comme « opérateurs cognitifs » du changement
L'utilité des protocoles ne peut évidemment, après le bilan effectué en première partie, qu'être mise en doute. Certes, l'emploi du principe de contractualisation pour réduire les arriérés constitue un pas important dans la perspective d'une rationalisation managériale de la gestion de l'institution judiciaire. Le message adressé aux chefs de corps est clair: l'octroi de cadres supplémentaires n'est plus gratuit. Le principal apport du dispositif n'est toutefois selon nous pas à chercher dans sa dimension formelle, mais dans sa fonction implicite: comme d'autres outils gestionnaires appliqués dans les juridictions belges, le conseiller en ressources humaines est avant tout ce que Denis Segrestin nomme un " opérateur cognitif » (2004, p. 29), dont l'utilité réformatrice est à évaluer à l'aune de sa capacité à " introduire une réflexion sur la gestion » (selon la formule d'un ancien conseiller de Laurette Onkelinx) et à favoriser sur le long terme l'appropriation de schémas de pensée et de modes de raisonnement managériaux par les personnels des parquets et tribunaux.

L'hypothèse posée ici est que la politique de modernisation de la Justice en Belgique, confrontée à la résistance des structures de l'institution, a favorisé la mise en place de dispositifs de gestion inspirés du secteur privé et tendant au développement d'un "sens commun réformateur " (Vauchez, Villemez, p. 239) parmi les magistrats et greffiers et à la mobilisation de ceux-ci autour d'objectifs d'accroissement de la productivité et de la qualité du service public judiciaire - en dépit des réticences que peut susciter un tel langage dans l'univers professionnel de la Justice.

Socialiser, convaincre, engager : la fonction implicite des conseillers en gestion des ressources humaines

Il convient donc, pour dépasser la description des tâches assumées au quotidien par les conseillers opérée en première partie, de relever la " fonction implicite » de leur présence dans les juridictions. On s'appuie ici sur les observations de David Courpasson, selon lequel «le management actuel se caractérise autant par la multiplication d'efforts de normalisation implicite, 'invisible', que par des efforts de contractualisation formelle » (1996, p. 239). Les recettes managériales tendraient ainsi à modifier les représentations et valeurs professionnelles de l'ensemble des acteurs de l'unité de production, à les engager au service de programmes de réforme des processus de travail et, en définitive, à produire collectivement de nouveaux modèles de travail légitimes aux yeux de tous: "Le management semble bien être, dans ses développements actuels, l'inscription de croyances et de convictions partagées dans un appareillage gestionnaire visant à réifier des représentations en règles, c'est-à-dire à normaliser » (Courpasson, 1996, p. 250). Ce processus de normalisation est censé susciter l'adhésion des acteurs aux objectifs de l'entreprise (ou, dans le cas présent, du service public) et accroître la productivité de cette dernière. De même, au travers de la mise en place 
La modernisation de la justice comme pédagogie du changement : le cas de l'intégration de "conseillers en gestion des ressources humaines " dans les juridictions belges

des conseillers en ressources humaines, il s'agit avant tout d'obtenir une évolution des mentalités, une redéfinition de la culture professionnelle des acteurs de la Justice par la sensibilisation à la logique managériale. Les conseillers eux-mêmes sont conscients de cette dimension cognitive sous-jacente :

"Donc, c'est une histoire de culture finalement : il faut changer la culture, c'est le plus difficile. Changer la façon de travailler ou changer ... certaines attitudes, c'est beaucoup plus facile, mais la culture, c'est vraiment l'âme, et changer l'âme, ça c'est beaucoup plus difficile (...) Mais, ça a quand même changé dans les têtes des magistrats" (entretien, conseiller en ressources humaines).

Un tel changement passe essentiellement par la persuasion et la délibération. Les avis et recommandations du conseiller, même s'ils ne sont pas suivis, contribuent à transformer la façon dont les acteurs définissent leurs problèmes, à désamorcer les réticences des personnels vis-à-vis des méthodes managériales, voire à susciter des initiatives de réforme.

" Je vois les personnes, ils m'expliquent ce qui ne va pas, et avec ma candeur, comme dirait le Procureur, je dis 'ça, ça ne va pas, il faudrait changer'. Et on discute ensemble des propositions d'amélioration. Et là, depuis que je suis ici, il y a des questions qu'avant on n'osait pas poser, des sections où ça ne fonctionnait pas mais on disait 'ça ne fonctionne pas mais comme on ne regarde pas c'est pas grave', et bien là, on y va, on se jette dans la fourmilière en disant 'qu'est-ce qu'on peut faire pour que ça marche ?' [...] Mais on a encore du retard sur l'effectivité du système. II y en a où c'est difficile, franchement difficile, mais où ça bouge, c'est le principal » (entretien, conseiller en ressources humaines).

"La vue du GRH nous permet d'apercevoir des problèmes qui nous échappent ou qui sont tellement évidents que nous n'y portons plus attention. C'est pour ça qu'il est bon d'avoir un œil extérieur » (entretien, Premier président de Cour d'Appel).

Les initiatives et recommandations de ces nouveaux acteurs semblent ainsi avoir abouti à l'intériorisation de certains impératifs gestionnaires, tel que celui du développement d'une véritable politique des ressources humaines. II ressort en effet des entretiens menés auprès des chefs de corps que les suggestions spontanées émises par leurs conseillers en faveur d'une rationalisation de la politique de formation ou d'une objectivation des critères de recrutement et d'évaluation ont modifié leur représentation du problème que posaient à eux la démotivation et le manque de compétence des personnels administratifs d'une part, des solutions à y apporter d'autre part. Dans les cas des Cours de Liège et Bruxelles, les Premiers présidents ont d'ailleurs entrepris d'étendre la démarche de rationalisation aux personnels assumant plus directement les tâches d'énonciation du droit (notamment les référendaires et juges de complément). Cette appropriation par les responsables des juridictions des idées initialement diffusées sous la forme de projets concrets par les 
conseillers en ressources humaines nous paraît illustrer la dimension pédagogique de l'action de ces derniers.

Le travail sur les croyances peut avoir un caractère plus volontariste lorsque le conseiller parvient à mettre en place un dispositif institutionnalisant la délibération autour d'enjeux de gestion, les acteurs de la juridiction étant alors incités à se positionner face au discours managérial et à réévaluer leurs attitudes. Le cas d'une démarche qualité initiée à la Cour d'appel de Bruxelles a été mentionné plus haut ; on peut également citer une initiative du conseiller auprès du Parquet de Bruxelles, qui a proposé de mettre en place un groupe représentant les différentes composantes du corps pour débattre collectivement des valeurs et formuler au nom de l'ensemble du personnel une "vision » partagée du parquet. II s'agit là de manière explicite de susciter un cadre normatif commun " officiel » afin de mobiliser les énergies autour des objectifs de l'organisation.

Les valeurs ainsi objectivées peuvent en effet être invoquées pour stimuler la productivité ou légitimer les changements; dans l'idéal, elles devraient être codifiées sous forme d'une charte approuvée par tous (le parquet de Louvain a ainsi, à l'initiative de son procureur, élaboré un tel document qui est affiché dans chaque service). Socialiser, convaincre puis engager : c'est en ces termes que pourrait se résumer la fonction des conseillers. Toutefois, du fait de leur situation marginale et des contraintes contextuelles qui s'imposent à eux, leur action a surtout pour objet de familiariser les acteurs de la Justice aux principes du management public et à les préparer à des réformes de plus grande envergure. L'efficacité d'un tel travail d'acculturation ne pourra probablement être correctement évaluée qu'à long terme, et en relation avec l'étude d'autres dispositifs aux fonctions similaires.

En effet, la création des conseillers en gestion des ressources humaines est exemplaire de la façon dont la politique de modernisation est pensée et menée en Belgique. De fait, les autres expérimentations managériales impulsées par le SPF Justice sont également conçues comme des vecteurs de modification des mentalités et de socialisation au management. Outre les conseillers, deux outils peuvent illustrer ce constat : la mise en place de cercles de qualité dans plusieurs « parquets pilotes " à partir de 2006 et les réflexions autour du développement d'un instrument de mesure de la charge de travail ; le dernier point revient brièvement sur ces deux enjeux, non pour en fournir une analyse exhaustive (qui dépasserait de loin le cadre de la présente étude), mais pour mieux faire ressortir par comparaison l'objectif sous-jacent de familiarisation aux solutions managériales qui unit les divers axes de la politique de modernisation de la Justice en Belgique.

\section{L'expérimentation managériale comme outil de modernisation de la Justice}

La promotion de la qualité de la Justice, comprise au sens de satisfaction des " usagers-clients", s'est imposée depuis une décennie comme but essentiel des réformes judiciaires en Belgique comme en Europe (Fabri et Langbroek, 2000 ; Breen, 2002 ; Jean et al., 2005). C'est ainsi que 
La modernisation de la justice comme pédagogie du changement : le cas de l'intégration de "conseillers en gestion des ressources humaines " dans les juridictions belges

l'obligation de veiller à la "qualité totale " des prestations des parquets est inscrite à l'article 7 de la loi du 4 mai 1997 sur l'intégration verticale du ministère public, qui définit les missions des Procureurs généraux. Plusieurs années de réflexion ont abouti à l'élaboration d'un protocole d'auto-évaluation des prestations du ministère public par les personnels des différents parquets, selon une grille de critères adaptée du modèle CAF (Common Assessment Framework) par l'Université Catholique de Louvain (Bouckaert, Depré, Drumeaux, 2006 ; Ficet, 2008a).

Cet exercice est explicitement conçu comme un moyen de développement de nouvelles compétences relationnelles et attitudes professionnelles. II s'agit de préparer l'avènement d'un nouveau rapport des employés à l'organisation en les confrontant à une situation inédite de communication horizontale et de débat autour de notions nouvelles. Les critères proposés aux évaluateurs reposent sur une échelle de valeurs qui ne leur est pas familière et, par les thématiques qu'elle désigne comme pertinentes et le système de notation qu'elle impose, la démarche qualité contribue à naturaliser des rôles gestionnaires et des standards organisationnels similaires à ceux que promeuvent, parallèlement, les réformes judiciaires en cours. II leur est ainsi demandé d'apprécier la qualité du leadership du chef de corps, la clarté de la "vision » et des valeurs qui inspirent l'action collective, le niveau de concertation interne et de motivation des personnels, la qualité de la communication avec les «partenaires » externes (police, avocats, municipalités...), l'écoute du client, l'inventivité dans l'amélioration des processus de travail, etc.

La démarche qualité promeut ainsi un idéal organisationnel fondé sur l'autonomie, la souplesse, la proactivité et la réflexivité ; elle constitue une sorte de pédagogie pratique de la communication et de l'auto-analyse, un vecteur de transmission des vertus managériales d'autant plus efficace qu'elle se donne à voir comme un instrument objectif d'amélioration du service au justiciable et qu'elle repose sur un engagement volontaire de ses participants. De tels dispositifs, s'ils n'ont été mis en œuvre à l'heure actuelle que dans quelques corps, sur proposition du SPF Justice ou à l'initiative des acteurs locaux (au parquet de Louvain et à la Cour d'appel de Bruxelles, notamment), sont amenés à se généraliser. II serait néanmoins particulièrement malaisé d'évaluer leur influence exacte sur les représentations professionnelles du corps judiciaire, les innovations managériales de ce type ne constituant à l'heure actuelle que des expériences exceptionnelles, souvent entravées par les contraintes organisationnelles propres à l'institution.

Un autre chantier de réformes en cours dans les tribunaux belges est la mesure de la charge de travail des magistrats (Connings et al., 2007). II a été mentionné plus haut que le Code judiciaire prévoit depuis une loi du 29 novembre 2001 que toute extension du cadre des magistrats d'un tribunal ou d'un parquet dépendra désormais d'une évaluation "reposant sur la mesure, au moyen d'un système d'enregistrement uniforme, de la charge de travail ». II s'agit, selon une pratique empruntée au management privé, de rationaliser l'emploi des ressources humaines par le biais d'un instrument "objectif " de quantification du temps consacré à chaque activité. Véritable serpent de mer de la modernisation de la 
Justice, l'enjeu de la mesure de la charge de travail a suscité de nombreux rapports et expérimentations, pour des résultats pour l'instant assez aléatoires (bien qu'un modèle développé par la firme de consulting Möbius soit à l'heure actuelle utilisé dans quelques parquets). Toutefois, à nouveau, l'essentiel n'est pas ici le résultat mais le processus de mise en œuvre d'un instrument managérial, au cours duquel les acteurs sont invités à s'approprier des normes professionnelles qui leur sont étrangères. Ainsi, à l'initiative de la Conférence des Premiers présidents de Cour d'appel, les magistrats des cinq Cours d'appels du pays ont entre février 2002 et juin 2004 rempli des "time sheets » indiquant, outre le type de contentieux, le degré de difficulté des affaires, le nombre de pièces incluses dans chaque dossier, le temps passé pour chaque étape de la procédure (préparation, audience, rédaction, prononcé...) (Castin, 2005 ; Hubin, 2008).

L'objectif officiel de l'expérience, inspirée par la méthode hollandaise Lamicie, était d'identifier le temps moyen accordé à chaque catégorie de dossiers. Mais au-delà, il s'agissait de sensibiliser les magistrats à l'enjeu de la réduction des arriérés et de légitimer auprès d'eux une approche quantitative des contentieux souvent critiquée comme incompatible avec " l'art » du verdict judiciaire. L'effet principal de l'expérience est dans cette perspective d'amener les juges à accepter la norme d'efficience comme critère d'excellence du travail juridictionnel. D'autres dispositifs participent d'ailleurs à cette intériorisation de l'impératif quantitatif, telle que la fixation d'objectifs chiffrés de production d'arrêts par chambre de jugement (système notamment adopté à la Cour d'appel de Bruxelles).

La mise en place des conseillers en ressources humaines n'apparaît donc pas comme une expérience isolée. Elle est exemplaire d'une politique de modernisation dont la cohérence ne provient pas tant de la complémentarité et de la systématicité des dispositifs mis en oeuvre que des valeurs qui les sous-tendent et de leurs objectifs implicites communs: la transmission d'un référentiel managérial et la mobilisation des personnels de la Justice autour d'une nouvelle représentation de l'activité juridictionnelle.

Conclusion L'hypothèse posée ici est donc que les conseillers contribuent avant tout à un changement de nature cognitive, ouvrant malgré l'étroitesse de leur marge de manœuvre opérationnelle la voie à une appropriation au niveau « micro » des méthodes managériales par les acteurs judiciaires. II nous a donc importé dans ce texte de saisir dans quelle mesure la présence des conseillers en gestion des ressources humaines participe (même faiblement) à la mutation des représentations professionnelles des praticiens de la Justice au contact desquels ils travaillent quotidiennement.

Au-delà du cas d'étude présent, la grille de lecture offerte par Segrestin, selon laquelle "les initiatives managériales méritent l'attention dès lors qu'elles font surgir de nouveaux cadres de délibération " (2007, p. 28), nous a paru pertinente dans le cadre de l'évaluation de la politique de modernisation de l'institution judiciaire en Belgique, fondée plus sur des dispositifs locaux que sur des réformes structurelles. 
La modernisation de la justice comme pédagogie du changement : le cas de l'intégration de "conseillers en gestion des ressources humaines " dans les juridictions belges

En effet, les réformes de l'Etat en général et de la Justice en particulier ont toujours été entravées par la nature consensuelle et consociative du système politique belge (Lijphart, 1981; Delwit, de Waele, Magnette, 1999) ; le vaste plan de managérialisation et de dépolitisation de l'administration fédérale engagé au début des années 2000 (Plan Copernic) a d'ailleurs de l'avis général abouti à un échec (de Visscher, 2005). Or, l'inertie liée à l'interpénétration entre administration et "piliers » est encore redoublée dans l'ordre judiciaire du fait des garanties constitutionnelles dont bénéficie la magistrature, associée de facto à toutes les décisions concernant l'organisation judiciaire. La mise en œuvre top-down d'un dispositif global de contrôle de la performance telle que la LOLF en France (Marshall, 2008) y serait de ce fait inimaginable.

II nous apparaît donc que, en l'attente d'une véritable décentralisation des moyens de gestion au niveau des juridictions qui mettrait la magistrature en demeure de se plier aux règles de la Nouvelle gestion Publique, la démarche pédagogique et non hiérarchique qu'illustre le dispositif des conseillers en ressources permet d'initier au sein de ce corps indépendant un processus d'acquisition des réflexes managériaux et de préparer à terme une mutation plus large des attitudes professionnelles des magistrats.

\section{BIBLOGRAPHIE}

BERRY M. (1983), Une technologie invisible? L'impact des outils de gestion sur les systèmes humains, Centre de recherche en gestion de l'Ecole Polytechnique.

BOUCKAERT G., DEPRE G. et DRUMEAUX A. (2006), Un instrument d'amélioration de la qualité dans le ministère public, Anvers, Maklu.

BOUCKAERT G. et POLLITT C. (2004), Public Management Reform. A Comparative Analysis, Oxford, Oxford Press University.

BREEN E. (dir.) (2002), Evaluer la Justice, Paris, PUF.

CASTIN M. (2005), " Initiatives récentes en matière de mesure de charge de travail dans les cours d'appel », dans DEPRE R., HONDEGHEM A. et PLESSERS J. (dir.), Managementhervormingen in Justitie, Die Keure, pp. 266-287.

CONNINGS V., DELVAUX D., DEPRE R., HONDEGHEM A. et MAESSCHALCK J. (2007), Etude de faisabilité de la mise en œuvre d'un instrument de mesure de la charge de travail destiné au siège, Recherche effectuée dans le cadre du programme "Action en soutien aux priorités stratégiques du Gouvernement », mis en œuvre par la Politique scientifique fédérale et le SPF Justice.

COURPASSON D. (1996), "Les normalisations managériales entre l'individu et le modèle professionnel », Revue d'économie industrielle, $\mathrm{n}^{\circ} 75$, pp. $239-256$. 
CROZIER M. et FRIEDBERG E. (1992), L'acteur et le système, Paris, Seuil.

DELWIT P., DE WAELE J.-M. et MAGNETTE P. (1999), Gouverner la Belgique: clivages et compromis dans une société complexe, Paris, PUF.

DEPRE R., HONDEGHEM A. et PLESSERS J. (2008), Le profil $d u$ " manager administratif " dans un contexte de modernisation de la Justice, Bruxelles, SPF Justice.

DE VISSCHER C. (2005), « Le coup dans l'eau de Copernic : réforme de la haute fonction publique, nouvelle gestion publique et particratie en Belgique », Politiques et management public, $\mathrm{n}^{\circ}$ 4, pp. 33-51.

DE VISSCHER C. et VARONE F. (2004), « La Nouvelle Gestion Publique 'en action' ", Revue internationale de politique comparée, $\mathrm{n}^{\circ} 2$, pp. 177 185.

FABRI M. et LANGBROEK P. (2000), The Challenge of Change for Judicial Systems, Amsterdam, IOS Press.

FICET J. (2008), «Les ambiguïtés de la gouvernance judiciaire. Autorégulation et qualité dans le ministère public belge ", Gouvernance, $\mathrm{n}^{\circ} 1$, (http://revuegouvernance.ca/files/Spring2008/Ficet.PDF).

FICET J. (2008), «La managérialisation de la fonction de chef de corps dans la magistrature belge: un état des lieux sociologique », dans Schoenaers F. et Dubois C. (dir.), Regards croisés sur le nouveau management judiciaire, Liège, Editions de l'Université de Liège, p. 57-81.

FRIEDBERG E. (1993), Le pouvoir et la règle, Paris, Seuil.

GOW J. et DUFOUR C. (2000), "Is the New Public Management a Paradigm ? Does It Matter ? ", International Review of Administrative Sciences, $n^{\circ} 4$, pp. 573-597.

HOOD C. (1991), "A Public Management For All Seasons ? ", Public Administration, $\mathrm{n}^{\circ} 1$, pp. 3-19.

HUBIN J. (2007), « Entre efficience et efficacité. Mesure et démesure de la charge de travail des magistrats du siège ", Pyramides, $\mathrm{n}^{\circ} 1$, pp. 100-146.

HUGHES E. (1958), Men and Their Work, Free Press, Glencoe.

JEAN J.-P., LANGBROEK P., PAULIAT H. (dir.) (2005), L'administration de la Justice en Europe et l'évaluation de sa qualité, Paris, Montchrestien.

LAENENS J. et STORME M. (dir.) (2000), Dans l'encre d'Octopus, Bruxelles, Bruylant.

LASCOUMES P. et LE GALES P. (2004), Gouverner par les instruments, Paris, Presses de la FNSP.

LIJPHART A. (dir.) (1981), Conflict and Coexistence in Belgium. The Dynamics of a Divided Society, Berkeley, University of California Press.

MARSHALL D. (2008), "L'impact de la Loi organique sur les lois de finances (LOLF) sur les juridictions », Revue française d'administration publique, $\mathrm{n}^{\circ} 1$, p. 121-131.

MAUGERI S. (dir.) (2006), Au nom du client. Management néolibéral et dispositifs de gestion, Paris, L'Harmattan. 
La modernisation de la justice comme pédagogie du changement : le cas de l'intégration de "conseillers en gestion des ressources humaines » dans les juridictions belges

METZGER J.-L. et BENEDETTO-MEYER M. (2008), Gestion et sociétés. Regards sociologiques, Paris, L'Harmattan.

MICHAUD C. et THOENIG J.-C. (2001), Stratégie et sociologie de l'entreprise, Paris, Village Mondial.

MINTZBERG H. (1990), Le management. Voyage au cœur des organisations, Paris, Les Editions d'Organisation.

SANCKEN S. (2001), " Justice et société : une justice vitrine en réponse à une société en émoi? : L'exemple de la Belgique des années 1980 et 1990 », Sociologie et sociétés, vol. 33, n 1, pp. 107-137.

SCHOENAERS F. (2003), Disponibilité des ressources et innovations managériales: quelles mutations pour les juridictions du travail belges et françaises face aux évolutions de leur environnement, thèse pour le doctorat en sociologie, IEP de Paris et Université de Liège.

SEGRESTIN D. (2007), Les chantiers du manager, Paris, Armand Colin.

VAUCHEZ A. et WILLEMEZ L. (2007), La Justice face à ses réformateurs (1980 -2006), Paris, PUF.

VERDUSSEN M. (dir.) (1999), Le Conseil supérieur de la Justice, Bruxelles, Bruylant.

VIGOUR C. (2004), «Réformer la Justice en Europe. Analyse comparée des cas de la Belgique, de la France et de l'Italie ", Droit et Société, $n^{\circ}$ 56/57, pp. 291-325.

VIGOUR C. (2006), « Justice : I'introduction d'une rationalité managériale comme euphémisation des enjeux politiques ", Droit et société, $\mathrm{n}^{\circ} 63$ 64, pp. 425-455.

PYRAMIDES (2006), numéro spécial «Les réformes de la Justice. Thémis : problème ou solution ? », $n^{\circ} 12$. 